\title{
Dependence of Rainfall on Solar Activity Features
}

Mahesh Chandra Mathpal, Bimal Pande and Seema Pande*

Department of Physics (UGC-Centre of Advanced Study), Kumaun University, Nainital, India.

Email: pande.seema@yahoo.com.

\begin{abstract}
In the present paper, we have studied the statistical analysis between all India homogeneous rainfall (RF) with sunspot number (SN) during 1900-2014 (115-year period). We have calculated correlations coefficient of rainfall with sunspot number $(\mathrm{SN})$ for annual and seasonal months: January, February (JF); March April May (MAM); June July August September (JJAS) and October November December (OND) and we have obtained high correlation ranging between 0.75 to 0.95 . Our results show that rainfall is strongly influenced by sunspot number. Our study also indicates that occurrence of solar activity features play an important role for variability of rainfall.
\end{abstract}

Keywords: Solar activity, Sunspot number, Rainfall variability.

\section{Introduction}

The solar activity features: Sunspot number $(\mathrm{SN})$, Solar Flare (SF), Solar active prominence (SAP) etc. affects the Earth's atmospheric phenomena like rainfall variability, climate change etc. There have been various statistical studies between rainfall and solar activity features (Reddy et al.,1989, Mitra et al., (1991), Hiremath et al., (2004), Hiremath., (2006), Bhattacharya et al., (2005), Bankoti et al., (2010), Selvaraj et al., 2013). (Hiramath and Mandi, 2004) have amply demonstrated the influence of solar activity over the earth global climate and environment. Sunspot number show significant correlation with Indian rainfall activity for seasonal as well as annual data (Hiremath, 2006). (Bhattacharyya et al., 2005) found that the rainfall is high during high solar activity features. (Bankoti et al., 2010) made a statistical study between solar activity features and all India homogeneous rainfall during 1963-2006 and reported a significant correlation between them. (Selvaraj et al., 2013) presented a cycle to cycle analysis between sunspot number and all India rainfall and indicated rainfall is maximum when the sunspot numbers are minimum and vice-versa. A strong linkage exists between average number of sunspots and all Indian rainfall as analysed by (Tripathi et al., 2014). The aim of present study is analyse the solar activity and homogeneous rainfall for a large data set of 115 years.

\section{Data and Statistical Study}

We have used 115 year data from 1900-2014 of rainfall (RF) and sunspots number(SN). $\mathrm{RF}$ data is obtained from Indian Meteorological data center and $\mathrm{SN}$ are obtained from the following websites:

http://www.tropmet.res.in.

http://www.ngdc.noaa.gov/stp/space-

weather/solar-data/solar-indices/sunspotnumbers/

The coefficient of correlation can be calculated as

$$
\boldsymbol{R}^{2}=\frac{n\left(\sum r . s\right)-\left(\sum r\right)\left(\sum s\right)}{\sqrt{\left[n \sum r^{2}-\left(\sum r\right)^{2}\right]\left[n \sum s^{2}-\left(\sum s\right)^{2}\right]}}
$$

Where $r=R F s=S N \cdot n=$ number of data points. 

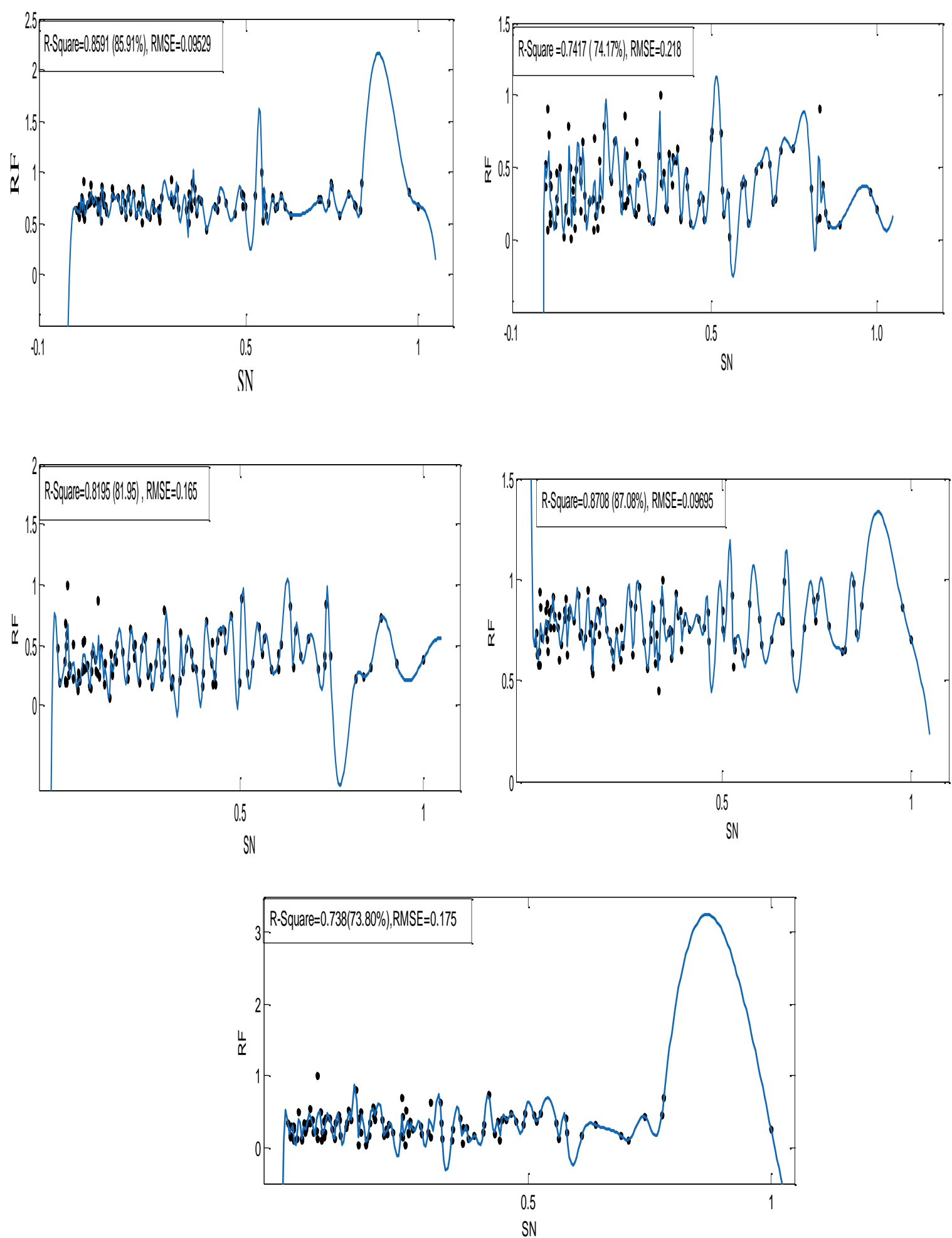

Fig.1. Coefficient of correlation of RF and SN and their RMSE for annual and Seasonal months 


\section{Results and Conclusions}

The correlation coefficient $\left(\mathrm{R}^{2}\right)$ value of $\mathrm{RF}$ with SN for annual and seasonal months (JF, MAM, JJAS, OND) are 0.85, 0.74, 0.81, $0.87 \& 0.73$ respectively. For check the significance of results here, we have applied chi-square test in which we have stated that the Null hypothesis's $\mathrm{H}_{0}$ : Rainfall independent of Sunspots numbers, alternative hypotheses $\mathrm{H}_{1}$ : Rainfall is not independent i.e. it is depends on solar activity features. We have calculated the $\chi^{2}$ values which are .000023 with 5 degrees of freedom for RF - SN. The statistical study between RF and $\mathrm{SN}$ indicate that the RF influenced by SN. According to seasonal and annual study we concluded that RF variation with $\mathrm{SN}$ is highly significant in summer (MAM) and monsoon season (JJAS) however, it shows less significant for winter season and annual RF. Hence, we can conclude that SN play an important role in rainfall variability and thereby climate change in the Earth's atmosphere. This confirms that solar phenomena are somehow Responsible for variation in Earth's atmospheric phenomena like rainfall, climate change etc. and these phenomena depends on solar atmospheric phenomena.

\section{References}

1. Bankoti, N. S., Joshi, N.C., Pande, S., Pande, B., Pandey, K., 2011, Quaternary International, 229, 8-15.

2. Bhatacharyya, S., Narasimha, R., 2005 Geophysical Research Letters,32, L05813,

3. Hiremath, K.M., Mandi, P.I., 2004., New Astronomy 9,651-652.

4. Hiremath., K.M., 2006, Journal of Astrophysics and Astronomy 27., 367-372.

5. Mitra, K., Mukherji, S., Dutta, S.N., 1991, International Journal of Climatology. 11, 645-652.

6. Selvaraj, R.S., Umarani, P.R., Mahalakshami. N., 2013 Journal of Geophysics Union., 17 (1), 59-63.
7. Tripathi K. D. Bhatacharyya A. B, 2014, International journal of Electronic and Communication technology., 3, 43-45. 